Review

\title{
Progesterone receptors - animal models and cell signaling in breast cancer Expression and transcriptional activity of progesterone receptor $A$ and progesterone receptor $B$ in mammalian cells
}

\author{
J Dinny Graham and Christine L Clarke
}

Westmead Institute for Cancer Research, University of Sydney Westmead Hospital, Westmead, New South Wales, Australia

Correspondence: Dinny Graham, Westmead Institute for Cancer Research, University of Sydney at Westmead Millennium Institute, Westmead Hospital, Westmead, New South Wales 2145, Australia. Tel: +61 29845 9066; fax: +61 29845 9102; e-mail: Dinny_Graham@wmi.usyd.edu.au

Received: 28 May

Accepted: 7 June 2002

Published: 2 July 2002
Breast Cancer Res 2002, 4:187-190

(C) 2002 BioMed Central Ltd

(Print ISSN 1465-5411; Online ISSN 1465-542X)

\begin{abstract}
Progesterone is an essential regulator of normal female reproductive function. Its effects are mediated by two nuclear progesterone receptor (PR) proteins, PRA and PRB, which are identical except for an additional 164 amino acids at the N-terminal end of PRB. Transcriptional analyses of the two receptor forms have assigned strikingly distinct functional signatures to the two PRs, despite their apparent physical similarity. The basis of these differences is yet to be fully understood. Furthermore, these differences are strongly influenced by the cell type and the promoter used. We review the mammalian transcriptional studies of PRA and PRB, and compare them with what is known about their expression and function in target tissues.
\end{abstract}

Keywords: progesterone receptor, progestin signaling, transcriptional activation

\section{Introduction}

Progesterone plays a pivotal role in normal female reproduction, in the uterus, the ovary, the mammary gland and the brain [1]. The number of cellular pathways regulated by progesterone reflects the complexity of its physiological role. In normal breast development, progesterone directs the formation of lobular-alveolar structures and also affects differentiation in the breast by modulation of milk protein synthesis [1]. In the human endometrium, progesterone directs glandular differentiation and glycogenesis, as well as stromal proliferation and development of predecidual cells [1]. These effects are mediated through the nuclear progesterone receptor (PR), expressed as two protein forms (PRA and PRB). There is increasing evidence to date that PRA and PRB are functionally different, and that it is the balance between these two forms that may make it possible for progesterone to affect such diverse physiological targets. Much of this evidence is conflicting and model specific, however, and the true differences between the receptor forms in normal tissues are yet to be fully understood.

The purpose of the present article is to review the current state of knowledge about the transcriptional differences between PRA and PRB from mammalian in vitro models, and to correlate this with the effects of altered PRA and PRB levels on target tissues.

\section{Transcriptional regulation by the PR}

The PR is a member of a large family of ligand-activated nuclear transcription regulators, which are characterised by organisation into specific functional domains and are conserved, to differing degrees, between species and family members. The PR is made up of a central DNA binding domain and a carboxyl-terminal ligand-binding domain. In addition, the receptor contains multiple activation function (AF) and inhibitory function elements, which enhance and 
repress transcriptional activation of the PR by association of these regions with transcriptional coregulators [2].

In most species examined, the PR exists as two distinct forms (PRA and PRB). The expression of human PR is controlled by two promoters, which direct the synthesis of two distinct subgroups of mRNA transcripts [3] encoding the two receptor proteins. The two PR forms are identical except that PRA lacks 164 amino acids contained at the $\mathrm{N}$ terminal end of PRB. The region of the protein that is unique to PRB contains a transcription activation function, AF3 [4], in addition to AF1 and AF2, which are common to PRA.

Newly transcribed cytoplasmic PR is assembled in an inactive multiprotein chaperone complex that dissociates on ligand binding and receptor activation. Progestin binding to the PR causes a conformational change and dimerisation, resulting in association of the progestincomplexed PR dimer with specific coactivators and general transcription factors. The activated complex binds to progestin response elements (PREs) in the promoters of target genes, resulting in modulation of transcription of those genes (reviewed in $[2,5]$ ).

There is now considerable evidence for differences in the transcriptional activities of PRA and PRB from transient cotransfection into a variety of cell lines of PRA and/or PRB and reporter constructs containing progestin-responsive sequences. These constructs range from the simple PRE-tk-CAT (containing one copy of a palindromic PRE) to more complex constructs such as those incorporating the mouse mammary tumor virus long terminal repeat, which contains multiple hormone-responsive elements.

PRB exhibits hormone-dependent transactivation in all cell types examined irrespective of the complexity of the response elements, whereas the transcriptional activity of PRA is cell specific and reporter specific. With reporter constructs containing a single palindromic PRE, PRA displays similar transactivation activity to PRB [6]. However, this activity is reduced or inactive when more complex response elements such as the mouse mammary tumor virus long terminal repeat and $\mathrm{PRE}_{2} \mathrm{TATA}_{\mathrm{tk}}$ constructs are used $[4,6,7]$. Interestingly, PRA acts as a transdominant inhibitor of PRB in situations where PRA has little or no transactivational activity $[6,7]$. Moreover, PRA can regulate the transcriptional activity of other nuclear receptors such as glucocorticoid, mineralocorticoid, androgen and estrogen [6-9], suggesting that PRA may play a central role in regulation of activity of a number of nuclear receptors in addition to PRB. The ability of PRA to act as a transdominant repressor is highly model specific, however, and there is considerable variability between reports.

McDonnell and Goldman [9] reported that PRA but not progestins, lessened the ability of estrogen to induce an estrogen-responsive reporter when the two constructs were transfected into CV-1 or HS578T cells, but not into HepG2 cells. PRA had similar anti-estrogenic effects on endogenous estrogen receptor activation of a minimal estrogen-responsive reporter in MCF-7 breast cancer cells in the presence of RU 38486 [8]. When the estrogen-responsive region of the pS2 gene was used as a reporter in MCF-7 cells, however, PRB and not PRA repressed activation of the reporter by estrogen [10].

The mechanisms by which PRA and PRB exert such apparently different transcriptional activities in various cell and promoter systems remain largely unknown, although a number of possible scenarios have been proposed. The physical differences at the $\mathrm{N}$-terminal end of the two receptors are clearly responsible for some transcriptional differences. In addition to the fact that AF3 is unique to PRB, the PRB-specific region has a distinct conformation in solution [11] and is likely to mask an inhibitory domain that is active in the N-terminus of the PRA protein [12]. This could act to enhance the transcriptional activity of PRB, as well as preventing it from acting as an inhibitor of other receptors.

The unique AF in PRB may confer a difference in affinities of the two PRs for coregulators. When compared in a phage display assay, the two PR forms bind to distinct subgroups of peptides. This suggests that coactivators may bind differently to the two PRs or that the two receptors bind to different subgroups of coactivators [13]. Motifs contained in AF3, with the same sequence as the NR boxes of coactivators, have been shown to be necessary for the transcriptional activity of the PRB-unique AF [14] and may form contacts between the receptor and a unique set of cofactors, or within the PR dimer itself. Given that the PR acts in combination with multiple other transcription factors to affect transcription, it is possible that variability of the tissue-specific expression of the components of this multiprotein complex may result in different PRA and PRB activities in the same cell. Furthermore, differential cofactor requirements between gene promoters may lead to differences in the transcriptional efficacy of the two PRs on the same promoter [2]. Further evidence is required to support these assertions since, although most cofactors are expressed at limiting levels, they have been demonstrated to be widely expressed throughout cell types. Furthermore, a degree of functional redundancy in PR coactivators seems to exist, with gene knockout studies of SRC-1 delivering only a mild phenotype [15] and the demonstration that TIF2 and SRC-1 are able to activate the PR to a similar extent in transfections [16].

The PR regulates the expression of a diverse population of transcriptional targets [1], and it would be expected that changes in the relative amounts of PRA and PRB would result in altered target gene expression patterns if the two 
isoforms are transcriptionally distinct. When patterns of gene regulation were examined in T47D breast cancer cells expressing exclusively PRA or PRB, a remarkably small overlap was seen between the sets of genes regulated by the two receptors, with the subset of genes regulated by PRB far exceeding in number those regulated by PRA [17]. When the relative expression of PRA and PRB was varied in wild-type T47D cells that already express both isoforms, however, the impact on transcription was not dramatic unless PRA was in vast excess over PRB [18]. Furthermore, no evidence was seen of dominant transcriptional inhibition by PRA. These data suggest that coexpression of both isoforms at similar levels, which is common in normal progesterone target cells (see later), is associated with appropriate transcriptional response to progestins and that changes in relative PRA and PRB levels must be quite dramatic before physiological changes in progestin signaling are observed.

\section{Expression and function of PRA and PRB in normal and malignant physiology}

In general, PRA and PRB are coexpressed in the same target cells in the human [19], and their relative expression, where it has been examined, is generally close to unity $[19,20]$. In some normal physiological circumstances, and in some cell types, there is a predominance of one isoform. PRA is always the predominant isoform in the uterine stroma, and PRB is predominantly expressed in the epithelial glands in the mid-secretory phase of the menstrual cycle [19]. PRA is the predominant isoform in the rodent [1] and is widely expressed in the macaque reproductive system.

Predominance of one isoform also occurs in cancers. In breast cancers, the equivalent expression of PRA and PRB seen in normal cells is disrupted early in carcinogenesis, and predominance of one isoform is common $[21,22]$. Most endometrial cancers express only one PR isoform, and isoform predominance is associated with higher histological grade [23]. The association of PR isoform predominance with cancer is supported by the demonstration that transgenic mice overexpressing PRA exhibited features in their mammary glands that were abnormal and commonly associated with neoplasia [24]. The expression of PRA and PRB in vivo supports a role for both isoforms in normal physiology, particularly in the human breast. The fact that the equivalent levels of the two proteins seen in the normal breast become disrupted early in breast carcinogenesis, and that predominance of one PR isoform, usually PRA, is seen in cancers, suggests that disrupted progesterone signaling may play a role in development or progression of breast cancer.

\section{Other mechanisms of PRA and PRB action}

In addition to the ligand-activated transcriptional effects already discussed, which reflect the nuclear activity of this receptor, the PR also regulates transcription via alternative pathways. Ligand-independent activation of the PR can occur and provides evidence for regulation of the PR via membrane-generated signals [25]. The PR also interacts with cytoplasmic signaling pathways to activate c-Src family members [26], and PR interactions with the mitogen-activated protein kinase pathway are also described. Migliaccio et al. found that PR activation of signaling pathways was independent of the transcriptional activity of the receptor and was indirectly mediated by PRB, but not PRA, through interaction with the estrogen receptor [26]. Other studies have examined the ability of the PR to transiently activate mitogen-activated protein kinase pathways but have generally focused on PRB, which was more transcriptionally active than PRA in most settings [27] (CA Lange, personal communication).

\section{Conclusion}

The in vitro data on the relative activities of PRA and PRB tend to support the view that PRB is the active PR, whereas PRA is either inactive or acts as an inhibitor of PRB activity. However, this perspective is at odds with the demonstrated coexpression of both isoforms in normal physiology $[1,19,20]$ and with the distinct roles ascribed to each protein from knockout studies discussed in the other sections of this review series. An explanation for the discrepancy between the in vivo and the in vitro data resides in the experimental protocols used to examine the question of relative PRA and PRB transcriptional activity. Most of the information has been obtained from transient transfection studies largely in cell lines that are not progestin targets, using exogenously transfected reporter sequences and each isoform in isolation. The relative levels of isoform protein coexpressed under these conditions cannot be known, and is probably highly relevant to interpretation of the data. There is little evidence in vivo that PRA is a dominant inhibitor of PRB. Moreover, when the data in human tissues and null animals are taken together, they suggest that the two isoforms either work cooperatively to mediate progesterone action or suggest that each isoform has distinct physiological roles that are probably cell specific and promoter specific. A combination of cooperative action and distinct activity is probably the best explanation for the complex and divergent pathways of progesterone action in normal and malignant physiology.

\section{Acknowledgements}

The authors are supported by grants from the National Health and Medical Research Council of Australia and the University of Sydney.

\section{References}

1. Graham JD, Clarke CL: Physiological action of progesterone in target tissues. Endocr Rev 1997, 18:502-519.

2. McKenna NJ, O'Malley BW: Combinatorial control of gene expression by nuclear receptors and coregulators. Cell 2002, 108:465-474.

3. Kastner P, Krust A, Turcotte B, Stropp U, Tora L, Gronemeyer H, Chambon P: Two distinct estrogen-regulated promoters generate transcripts encoding the two functionally different 
human progesterone receptor forms A and B. EMBO J 1990, 9:1603-1614.

4. Sartorius CA, Melville MY, Hovland AR, Tung L, Takimoto GS, Horwitz KB: A third transactivation function (AF3) of human progesterone receptors located in the unique $\mathrm{N}$-terminal segment of the B isoform. Mol Endocrinol 1994, 8:1347-1360.

5. Tsai M-J, O'Malley BW: Molecular mechanisms of action of steroid/thyroid receptor superfamily members. Ann Rev Biochem 1994, 63:451-486.

6. Tung L, Mohamed MK, Hoeffler JP, Takimoto GS, Horwitz KB: Antagonist-occupied human progesterone B-receptors activate transcription without binding to progesterone response elements and are dominantly inhibited by A-receptors. Mol Endocrinol 1993, 7:1256-1265.

7. Vegeto E, Shabaz MM, Wen DX, Goldman ME, O'Malley BW, McDonnell DP: Human progesterone receptor A form is a celland promoter-specific repressor of human progesterone receptor B function. Mol Endocrinol 1993, 7:1244-1255.

8. Wen DX, Xu Y-F, Mais DE, Goldman ME, McDonnell DP: The A and $B$ isoforms of the human progesterone receptor operate through distinct signaling pathways within target cells. Mol Cell Biol 1994, 14:8356-8364.

9. McDonnell DP, Goldman ME: RU486 exerts antiestrogenic activities through a novel progesterone receptor $A$ formmediated mechanism. J Biol Chem 1994, 269:11945-11949.

10. Chalbos D, Galtier F: Differential effect of forms A and B of human progesterone receptor on estradiol-dependent transcription. J Biol Chem 1994, 269:23007-23012.

11. Bain DL, Franden MA, McManaman JL, Takimoto GS, Horwitz KB: The N-terminal region of human progesterone B-receptors: biophysical and biochemical comparison to A-receptors. $J$ Biol Chem 2001, 276:23825-23831.

12. Huse B, Verca SB, Matthey P, Rusconi S: Definition of a negative modulation domain in the human progesterone receptor. Mol Endocrinol 1998, 12:1334-1342.

13. Giangrande $\mathrm{PH}$, Kimbrel EA, Edwards DP, McDonnell DP: The opposing transcriptional activities of the two isoforms of the human progesterone receptor are due to differential cofactor binding. Mol Cell Biol 2000, 20:3102-3115.

14. Tung L, Shen T, Abel MG, Powell RL, Takimoto GS, Sartorius CA, Horwitz KB: Mapping the unique activation function 3 in the progesterone B-receptor upstream segment. Two LXXLL motifs and a tryptophan residue are required for activity. $J$ Biol Chem 2001, 276:39843-39851.

15. Xu J, Qiu Y, DeMayo FJ, Tsai SY, Tsai MJ, O'Malley BW: Partial hormone resistance in mice with disruption of the steroid receptor coactivator-1 (SRC-1) gene. Science 1998, 279:19221925.

16. Onate SA, Boonyaratanakornkit V, Spencer TE, Tsai SY, Tsai MJ, Edwards DP, O'Malley BW: The steroid receptor coactivator-1 contains multiple receptor interacting and activation domains that cooperatively enhance the activation function 1 (AF1) and AF2 domains of steroid receptors. J Biol Chem 1998, 273: 12101-12108

17. Richer JK, Jacobsen BM, Manning NG, Abel MG, Wolf DM, Horwitz KB: Differential gene regulation by the two progesterone receptor isoforms in human breast cancer cells. $J$ Biol Chem 2002, 277:5209-5218.

18. McGowan EM, Clarke CL: Effect of overexpression of progesterone receptor $A$ on endogenous progestin-sensitive endpoints in breast cancer cells. Mol Endocrinol 1999, 13: 1657-1671.

19. Mote PA, Balleine RL, McGowan EM, Clarke CL: Colocalization of progesterone receptors $A$ and $B$ by dual immunofluorescent histochemistry in human endometrium during the menstrual cycle. J Clin Endocrinol Metab 1999, 84:2963-2971.

20. Mote PA, Balleine RL, McGowan EM, Clarke CL: Heterogeneity of progesterone receptors $A$ and $B$ expression in human endometrial glands and stroma. Hum Reprod 2000, 15:48-56.

21. Graham JD, Yeates C, Balleine RL, Harvey SS, Milliken JS, Bilous AM, Clarke CL: Characterization of progesterone receptor A and B expression in human breast cancer. Cancer Res 1995, 55:5063-5068.

22. Mote PA, Bartow S, Tran N, Clarke CL: Loss of co-ordinate expression of progesterone receptors $A$ and $B$ is an early event in breast carcinogenesis. Breast Cancer Res Treat 2002, 72:163-172.
23. Arnett-Mansfield RL, deFazio A, Wain GV, Jaworski RC, Byth K, Mote PA, Clarke CL: Relative expression of progesterone receptors $A$ and $B$ in endometrioid cancers of the endometrium. Cancer Res 2001, 61:4576-4582.

24. Shyamala G, Yang X, Silberstein G, Barcellos-Hoff MH, Dale E: Transgenic mice carrying an imbalance in the native ratio of $A$ to $B$ forms of progesterone receptor exhibit developmental abnormalities in mammary glands. Proc Natl Acad Sci USA 1998, 95:696-701.

25. Denner LA, Weigel NL, Maxwell BL, Schrader WT, O'Malley BW: Regulation of progesterone receptor-mediated transcription by phosphorylation. Science 1990, 250:1740-1743.

26. Migliaccio A, Piccolo D, Castoria G, Di Domenico M, Bilancio A, Lombardi M, Gong W, Beato M, Auricchio F: Activation of the Src/p21ras/Erk pathway by progesterone receptor via crosstalk with estrogen receptor. EMBO J 1998, 17:2008-2018.

27. Lange CA, Richer JK, Shen T, Horwitz KB: Convergence of progesterone and epidermal growth factor signaling in breast cancer. Potentiation of mitogen-activated protein kinase pathways. J Biol Chem 1998, 273:31308-31316. 